\title{
Effects of Chronic Treatment with Carvedilol on Smooth Muscle Tone and Endothelium-Dependent Relaxation of Aorta in Stroke-Prone Spontaneously Hypertensive Rats
}

\author{
Satoru Sunano, Fumiko Sekiguchi, Akiko Nakamura, \\ Kyoko Matsuda, Kazuo Yamamoto, Tomoko Shibutani*, \\ Hiroo Hashimoto*, Makoto TanaKA* and Keiichi Shimamura \\ Faculty of Pharmaceutical Sciences and Research Institute of Hypertension, \\ Kinki University, Osaka and ${ }^{*}$ Tokyo $R \& D$ Center, Daiichi Pharmaceutical \\ Co., Ltd., Tokyo, Japan.
}

\begin{abstract}
Effects of chronic treatment of stroke-prone spontaneously hypertensive rats (SHRSP) with carvedilol, an agent which has both $\alpha$ - and $\beta$-adrenoceptor blocking actions, on spontaneous muscle tone and on structural and functional abnormalities of endothelium were studied. The treatment of SHRSP with the drug of the dose of 30 to $200 \mathrm{mg} / \mathrm{kg} / \mathrm{day}$ lowered the blood pressure significantly. Spontaneous muscle tone in endothelium-removed preparation disappeared by the treatment. Noradrenaline-induced contraction was depressed by the treatment in endothelium-intact preparation but not in endotheliumremoved preparation. The treatment prevented the structural and functional abnormalities of endothelium. Similar results were obtained by the treatment with propranolol.

These results indicate that carvedilol prevented abnormal contraction of SHRSP aorta through protective effects on smooth muscle and endothelium. These effects may play roles in blood pressure lowering effect of carvedilol.
\end{abstract}

Key words: hypertensive rats, carvedilol, propranolol, aorta, endothelium, noradrenaline, smooth muscle tone

Abnormalities in vascular contraction have been reported in hypertension (Triggle and Laher, 1985). Aortic smooth muscle of spontaneously hypertensive rats, for example, shows spontaneous tension development (tone) which is rarely observed in normotensive rats (Noon et al., 1978 ; Sada et al., 1989a ; Sunano and Shimamura, 1991 ; Sasaki et al., 1993). The function of endothelium is also altered in the aorta of spontaneously hypertensive rats (Konishi and Su, 1983 ; Sugimoto et al., 1988 ; Sunano et al., 1989 ; Lüscher et al., 1991).

Chronic antihypertensive treatment with angiotensin converting enzyme inhibitors prevented functional abnormalities in vascular smooth muscle (Sada et al., 1989a, 1989b ; Sunano and Shimamura, 1991; Sunano et al., 1992) and endothelium (Clozel et al., 1990 ; Clozel, 1991;

Current address : Faculty of Pharmaceutical Sciences, Kinki University, 3-4-1 Kowakae, Higashi-Osaka, Osaka 577, Japan

Correspondence to: Dr. Satoru Sunano 
Sunano et al., 1992) of hypertensive rats. Impairment of endothelial function was prevented by chronic treatment with hydralazine (Sunano et al., 1989 ; Nigro et al., 1989 ; Shimamura et al., 1991 ; Sunano et al., 1993).

Antihypertensive treatment combination with other drugs of different mechanisms is considered to be effective because antihypertensive treatment via one mechanism leads to activation of other compensatory process. Recently multiple-action agents have been introduced for antihypertensive treatment (Rahnm, 1987 ; Prichar, 1992). Carvedilol is a $\beta-$ adrenoceptor blocking agent with $\alpha$ 1-adrenoceptor blocking activity (Sponer et al., 1987 ; Seki et al., 1988 ; Hashimoto et al., 1988,1991; McTavish et al., 1993). However, no report is available on the effect of chronic treatment of carvedilol on vascular function. In the present experiment, effects of chronic treatment with carvedilol on the tone of smooth muscle and endothelium-dependent relaxation in aorta of stroke-prone spontaneously hypertensive rats (SHRSP) were studied.

\section{Materials and Methods}

\section{Materials}

Stroke-prone spontaneously hypertensive rats (SHRSP) and normotensive Wistar Kyoto rats (WKY) were used in the present experiments. They were originally obtained from Dr. Okamoto (Okamoto et al., 1974) and bred successively in our animal facility under the conditions of $25^{\circ} \mathrm{C}, 50 \%$ humidity and light and dark cycle of 12 hours. Great care was taken in breeding the animal to maintain expected blood pressure. Systolic blood pressure of the rats was measured by means of tail cuff method. Prior to the measurement, the rats were warmed at $40^{\circ} \mathrm{C}$ for 5 minutes. They were sacrificed at age of 16 weeks under anesthesia with ethyl ether.

Treatments of hypertensive rats with carvedilol were started at the age of weaning (5 weeks old) and continued until the experiments ( 16 weeks old). The drug was mixed with chow changing the dose. The doses were in the range of $30 \mathrm{mg} / \mathrm{kg} /$ day (5 weeks old) to $200 \mathrm{mg} / \mathrm{kg} /$ day (16 weeks old). Treatment with propranolol was also performed giving the drug of 100 $\mathrm{mg} / \mathrm{kg} /$ day from 5 weeks to 16 weeks of age. Amount of drugs administered was estimated from drug-mixed chow weight consumed by rats. Further increment of the doses was not available because of losses of appetite and body weight.

Mechanical responses (contraction and relaxation) of blood vessel were observed using aortae of rats at 16 weeks old. Thoracic aorta was excised and ring preparations of $1 \mathrm{~mm}$ width were made. In about one third of the preparations, endothelium was removed by rubbing the inner surface with soft rubber band. These preparations were mounted in the organ bathes filled with a modified Tyrode's solution of following composition $(\mathrm{mM}): \mathrm{NaCl}$, $137 ; \mathrm{KCl}, 5.4 ; \mathrm{CaCl}_{2}, 2.0 ; \mathrm{MgCl}_{2}, 1.0 ; \mathrm{NaHCO}_{3}, 11.9 ; \mathrm{NaH}_{2} \mathrm{PO}_{4}, 0.4$; glucose, 5.6. The solution was kept at $37^{\circ} \mathrm{C}$ and aerated with a gas mixture of $95 \% \mathrm{O}_{2}$ and $5 \% \mathrm{CO}_{2}$. High-KTyrode's solution was made by replacing $\mathrm{NaCl}$ in the solution with equimolar $\mathrm{KCl}$, and $\mathrm{Ca}$-free Tyrode's solution, by omitting $\mathrm{CaCl}_{2}$ from the modified Tyrode's solution. 


\section{Methods}

Tension changes of the preparations were measured isometrically with a force-displacement transducer (Shinkoh, Japan) under stretch tension of $8 \mathrm{mN}$. Changes in stretch tension around this range, taking difference in the wall thickness of the preparations from SHRSP and WKY into consideration, did not show significant influences on the contraction of the both preparations. Endothelium-dependent relaxation, however, became weaker at higher stretch tension in both preparations. The experiments were started after two successive high-K(50 $\mathrm{mM}$ )-contractures. This procedure was required to obtain the constant results in the experiments.

Scanning electron microscopic observation of endothelium was performed as follows. Vena cava was cut under ethyl ether anesthesia and animals were perfused from left ventricle with the modified Tyrode's solution at pressure of $70 \mathrm{mmHg}$ for 10 minutes. The perfusing solution were then changed to $2.5 \%$ glutaraldehyde in phosphate buffer $\mathrm{pH}$ 7.4. Then, the aorta was dissected, opened lengthwise, and pinned on a Teflon sheet. The tissue was postfixed in $1 \%$ osmic acid $\left(\mathrm{OsO}_{4}\right)$ in $0.1 \mathrm{M}$ phosphate buffer at room temperature. The tissue was then dehydrated through graded ethanol, critical point-dried in $\mathrm{CO}_{2}$, and sputter-coated with goldpalladium and observed by scanning electron microscope (Hitachi S-800, Japan).

\section{Drugs}

Drugs used in the present experiment were; carvedilol (Daiichi, Tokyo, Japan), propranolol $\cdot \mathrm{HCl}$ (Sigma, St. Louis, USA), noradrenaline bitartrate (Sigma, St. Louis, USA), acetylcholine $\cdot \mathrm{HCl}$ (Wako, Osaka, Japan), papaverine $\cdot \mathrm{HCl}$ (Wako, Osaka, Japan), verapamil $\mathrm{HCl}$ (Ei-sai, Osaka, Japan) and $\mathrm{N}^{\mathrm{G}}$-nitro-L-arginine (Aldrich, Wisconsin, USA).

Obtained data were expressed as mean $\pm \mathrm{SE}$ and analyzed using Student's t-test, considering $\mathrm{P}$ values smaller than 0.05 to be statistically significant.

\section{Results}

Body weight of the rats.

Body weight of WKY and SHRSP at 16 weeks of age was $310.1 \pm 6.4 \mathrm{~g}(\mathrm{n}=29)$ and $259.4 \pm$ $4.0 \mathrm{~g}(\mathrm{n}=28)$, respectively. The body weight of WKY was greater than SHRSP $(\mathrm{p}<0.001)$. The body weight of the SHRSP treated with carvedilol and propranolol was $217.2 \pm 8.4 \mathrm{~g}(\mathrm{n}=$ $11)$ and $242.4 \pm 7.1 \mathrm{~g}(\mathrm{n}=10)$, respectively. The body weight of treated SHRSP was slightly smaller than that of untreated SHRSP $(\mathrm{p}<0.001$ in carvedilol-treated SHRSP and $\mathrm{p}<0.05$ in propranolol-treated SHRSP).

\section{Blood pressure of animals.}

Blood pressure of SHRSP at 5 weeks old was already significantly higher than that of WKY $(\mathrm{P}<0.001)$ and rose steeply with aging and reached at high plateau level in 12 weeks of age. The blood pressure at the age of 16 weeks was $245.8 \pm 2.9 \mathrm{mmHg}(\mathrm{n}=28)$. Rise of blood pressure of WKY with aging, on the other hand, was not prominent and the blood pressure at 16 weeks of age was $135.3 \pm 1.2 \mathrm{mmHg}(\mathrm{n}=29)$. The difference in the blood pressure between 
SHRSP and WKY at 16 weeks of age was significant $(\mathrm{p}<0.001)$.

Carvedilol lowered the blood pressure in 3 weeks after starting the treatment. The blood pressure of the carvedilol-treated and propranolol-treated SHRSP was $199.8 \pm 1.9 \mathrm{mmHg}(\mathrm{n}=$ 11) and $208.1 \pm 5.4 \mathrm{mmHg}(\mathrm{n}=10)$, respectively, being significantly lower than that of untreated SHRSP $(\mathrm{p}<0.05)$ at 16 weeks old of age (Fig. 1a, b).

\section{Spontaneous tension development}

Endothelium-removed preparation from 16 weeks old SHRSP exhibited spontaneous tension development (tone) in the absence of stimulants. The tone was dependent of extracellular $\mathrm{Ca}^{++}$. It disappeared by the removal of extracellular $\mathrm{Ca}^{++}$or by the application of verapamil $\left(10^{-6} \mathrm{M}\right)$. On the contrary, the addition of $\mathrm{Ca}^{++}$to $\mathrm{Ca}^{++}$-deprived medium induced tension development $\mathrm{Ca}^{++}$-concentration dependently. The tone was depressed by endothelium and only weak tone was observed in endothelium-intact preparation (Fig. 2a). In aortic preparation of WKY, little tone was observed in endothelium-intact or -removed preparations (Fig. 2b).

Effects of carvedilol-treatment on the tone of aortic smooth muscle was studied using endothelium-removed preparation of SHRSP. The treatment prevented the development of spontaneous tone. Only small tone was observed in endothelium-removed preparation from carvedilol-treated SHRSP (Fig. 3a). Similar effect was observed by the treatment of SHRSP with propranolol (Fig. 3b).

\section{Noradrenaline-induced contraction}

Noradrenaline induced concentration-dependent contraction both in the endotheliumintact and -removed preparations from SHRSP and WKY. The maximal contraction was achieved at $10^{-5} \mathrm{M}$ in the preparations both from SHRSP and WKY. No significant difference
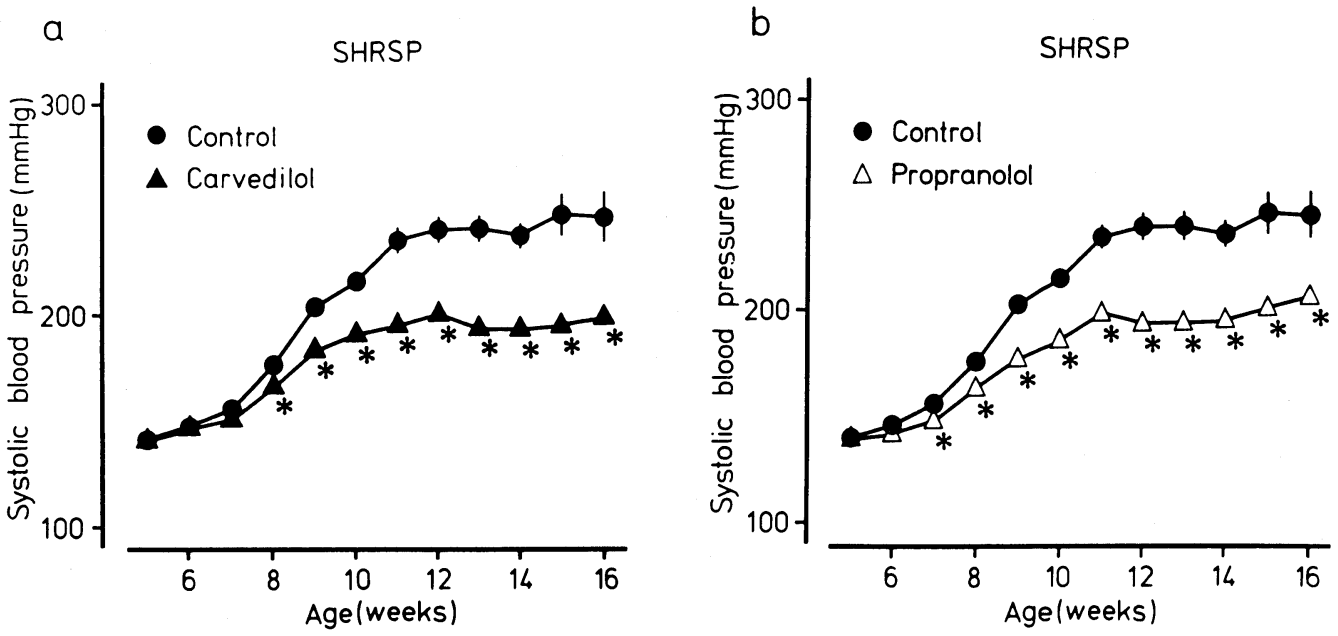

Fig. 1. Effects of treatment with carvedilol (a), and propranolol (b) on the systolic blood pressure of SHRSP. The treatment was started at 5 weeks of age and their doses were determined to keep blood pressure under $200 \mathrm{mmHg}$ at 16 weeks of age as described in the methods. Mean \pm SE of 10 rats, respectively. Asterisks indicate significant differences from untreated control rats $(\mathrm{P}<0.05)$. 
a

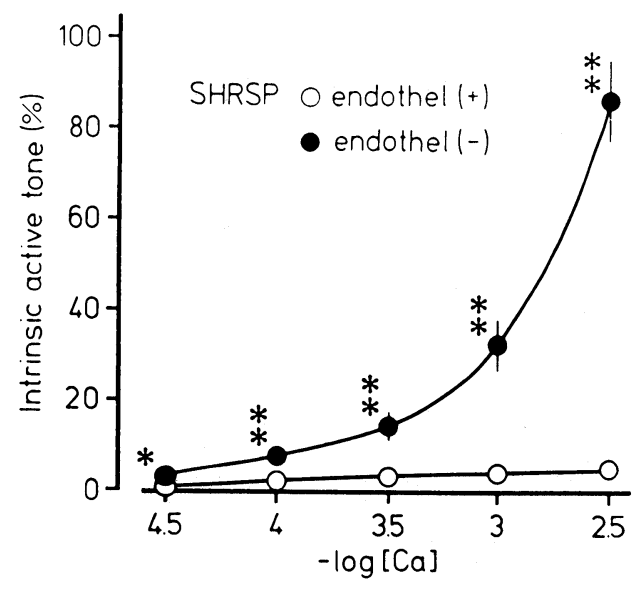

b

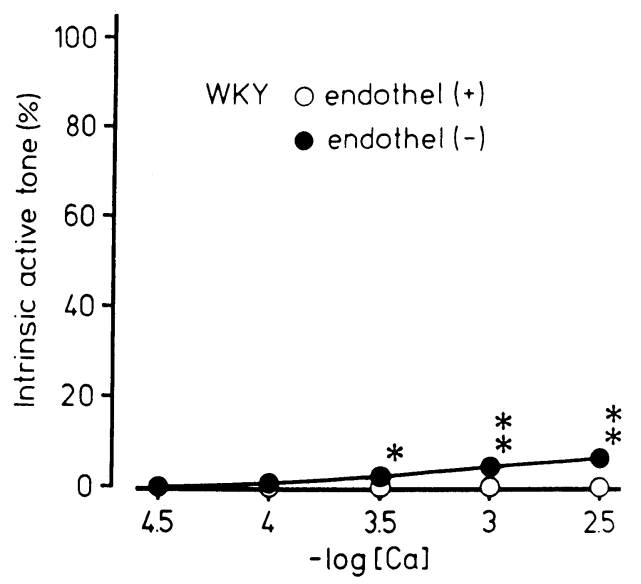

Fig. 2. Dependence of intrinsic active tone on extracellular $\mathrm{Ca}$ and its depression by endothelium in aorta from SHRSP (a) and WKY (b). endothel $(+)$ : endothelium-intact preparation $(n=6$ to 9$)$. endothel $(-)$ : endothelium-removed preparation $(n=20$ to 22$)$. Asterisks indicate significant differences from endothelium-intact preparation $\left(^{*}, \mathrm{P}<0.05{ }^{* *}\right.$, $\mathrm{P}<0.01)$.

a

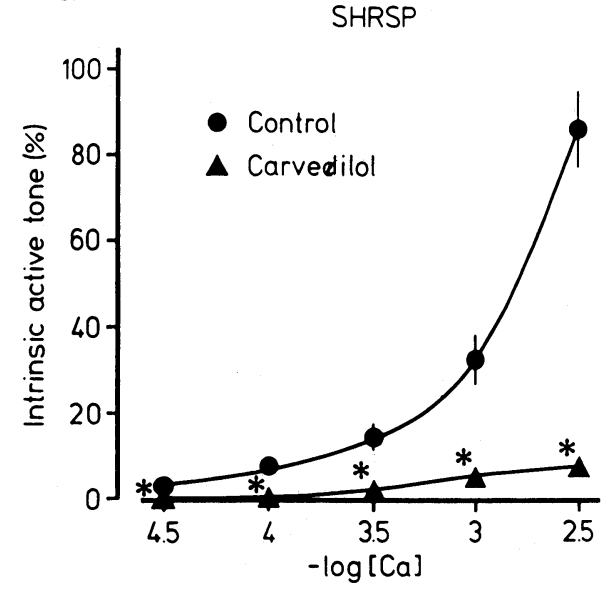

b

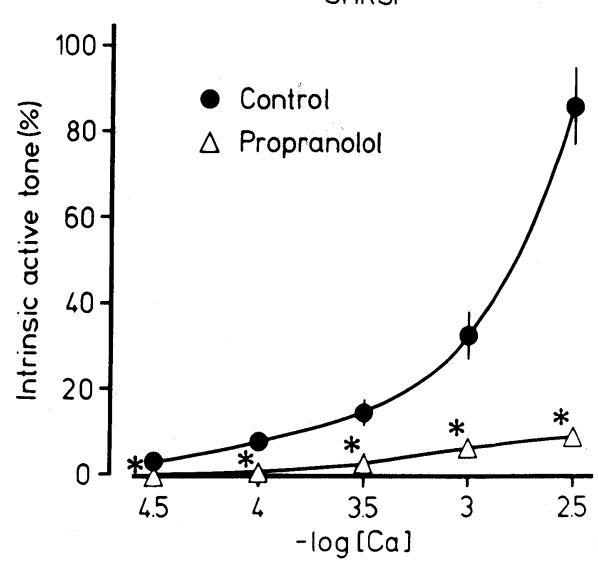

Fig. 3. Effects of treatment of SHRSP with carvedilol (a) and propranolol (b) on intrinsic active tone of endothelium-removed preparation at different extracellular $\mathrm{Ca}$ concentration. Note that both treatments depressed the active tone to similar level to that of WKY aorta shown in Fig. 2. $\mathrm{n}=20$ in untreated, 14 in carvedilol-treated and 11 in propranolol-treated rats, respectively. Asterisks indicate significant differences from control $(\mathrm{P}<0.01)$.

in the developed tension between these preparations was observed (Fig. 4).

Endothelium depressed noradrenaline-induced contraction markedly. Endothelium depressed the contraction in the preparation from WKY more prominently than SHRSP (Fig. 4).

In endothelium-intact preparation of SHRSP, carvedilol-treatment depressed the nora- 

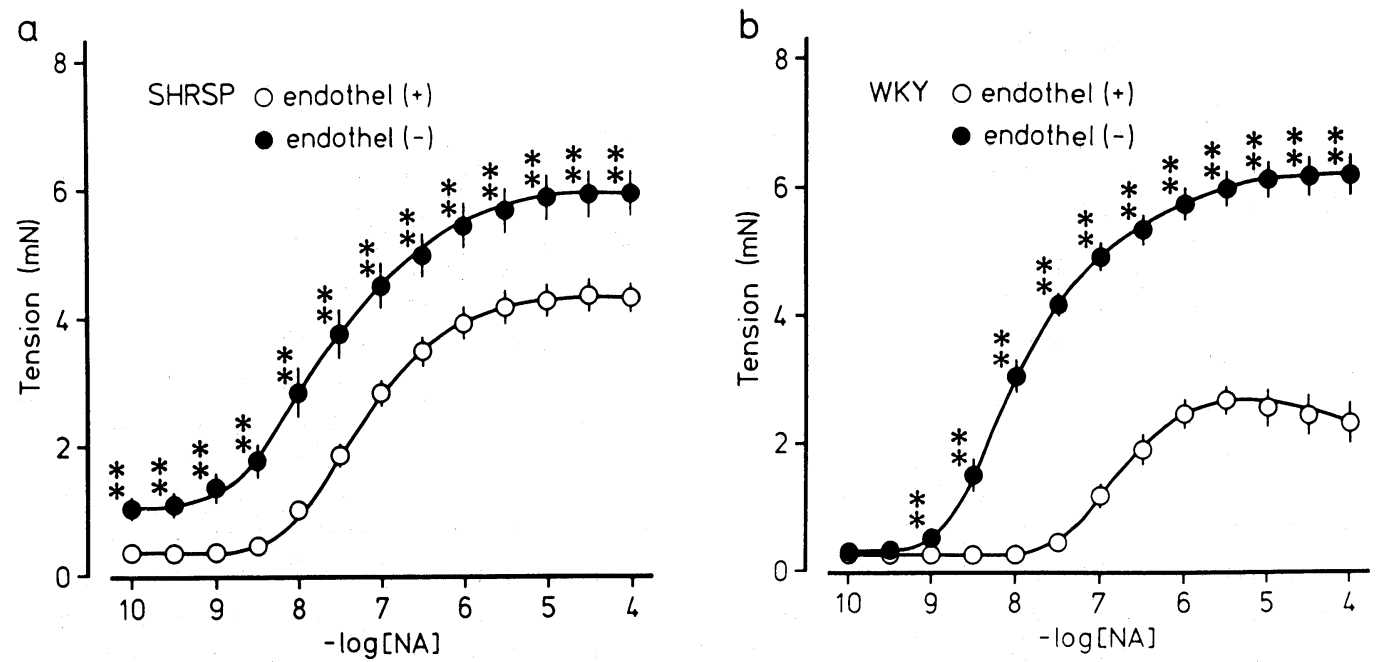

Fig. 4. Dose-response curve for noradrenaline-induced contraction in endothelium-intact and -removed preparation. (a) aorta of SHRSP. (b) aorta of WKY. endothel $(+)$ : endothelium-intact preparation. endothel $(-)$ : endothelium-removed preparation. $n=16,11,9$ and 13 in endothelium-intact, -removed preparation from SHRSP aorta and endotheliumintact, -removed preparation from WKY, respectively. Asterisks indicate significant differences from endothelium-intact preparation $(\mathrm{P}<0.01)$.

a

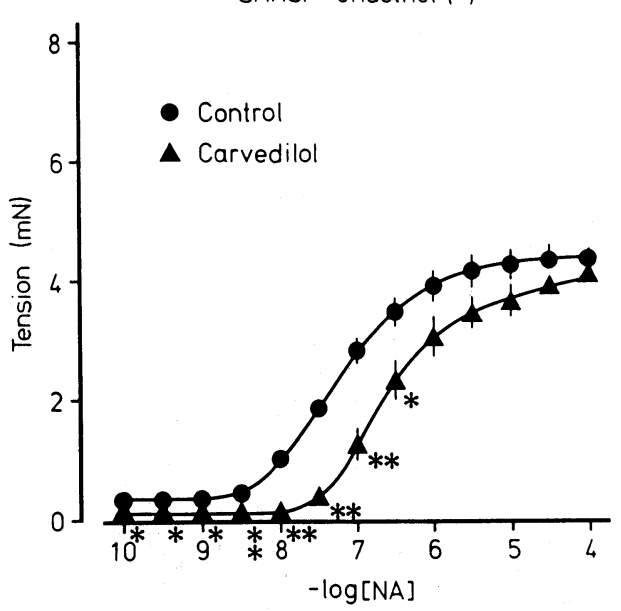

b

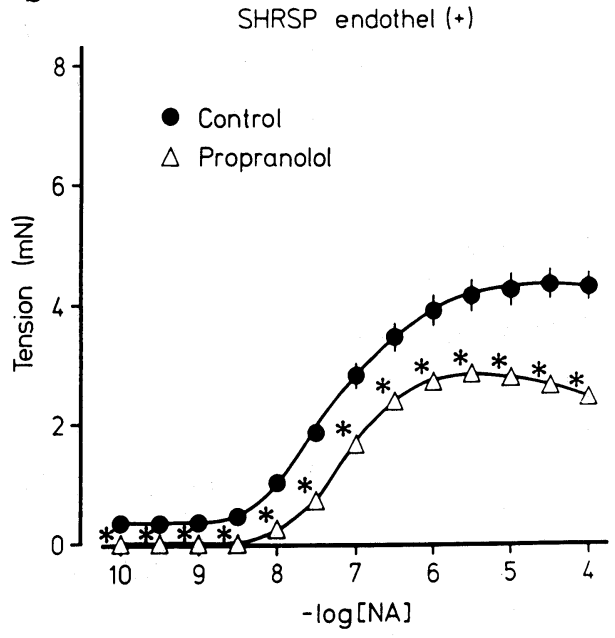

Fig. 5. Effects of treatment of SHRSP with carvedilol (a) and propranolol (b) on noradrenalineinduced contraction of endothelium-intact preparation. $\mathrm{n}=8$ and 11 in preparations from carvedilol- and propranolol-treated SHRSP, 16 in untreated control SHRSP. Asterisks indicate significant differences from preparations of untreated SHRSP $\left({ }^{*}, \mathrm{P}<0.05\right.$ **, $\mathrm{P}<0.01)$. 
drenaline-induced contraction and shifted the concentration-response curve to the right (Fig. 5a). Similar depression was observed by the treatment with propranolol. The depression of the contraction was greater than that in carvedilol-treatment (Fig. 5b).

Spontaneous tone of the preparation was depressed by the carvedilol or propranolol treatment. There was no remarkable effect of the treatments on noradrenaline-induced contraction in endothelium-removed preparation.

\section{High $-K^{+}-$induced contraction}

When $\mathrm{K}^{+}$-concentration of the Tyrode's solution was increased, aortic preparation contracted in a concentration-dependent manner. In endothelium-intact preparation, high- $\mathrm{K}^{+}-$

a

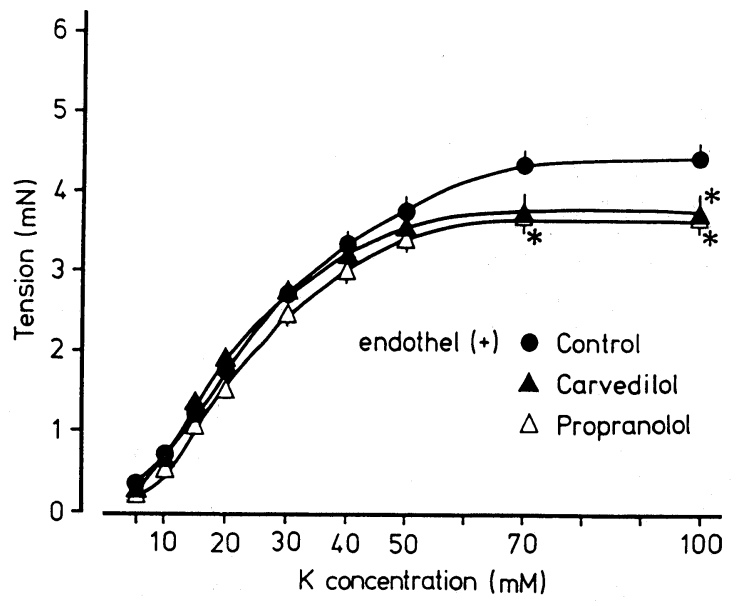

b

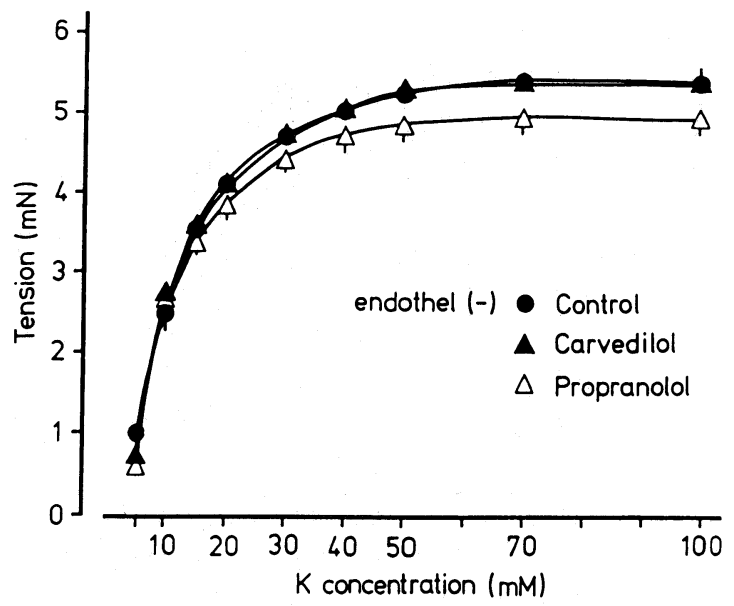

Fig. 6. Effects of treatment of SHRSP with carvedilol and propranolol on high- $\mathrm{K}^{+}$-induced contraction. (a) endothelium-intact preparation. (b) endothelium-removed preparation. Number of preparations for each point is between 12 and 25 . Asterisks indicate significant differences from untreated SHRSP $(\mathrm{P}<0.05)$.

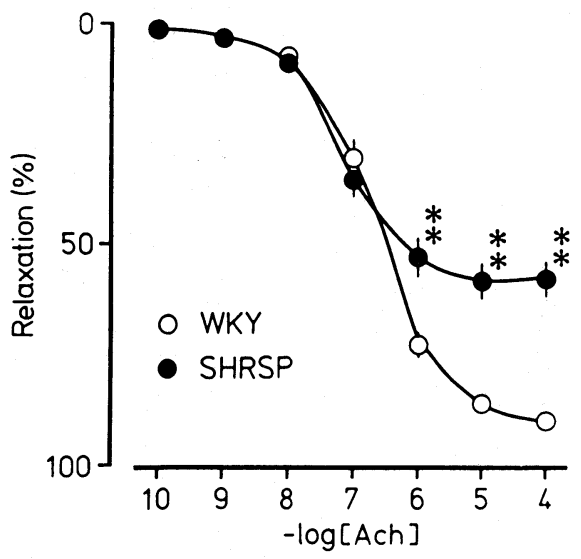

Fig. 7. Acetylcholine-induced endotheliumdependent relaxation of aortic preparation from SHRSP and WKY. Aortic preparations were precontracted by $5 \times$ $10^{-7} \mathrm{M}$ noradrenaline and, after the contraction achieved its maximum, acetylcholine was applied cumulatively. The relaxation by acetylcholine was expressed as percentages of the contraction induced by noradrenaline. $\mathrm{n}=21$ and 18 , in the preparation from WKY and SHRSP, respectively. Asterisks indicate significant differences from WKY $\left({ }^{* *}, \mathrm{P}<0.01\right)$. 
a

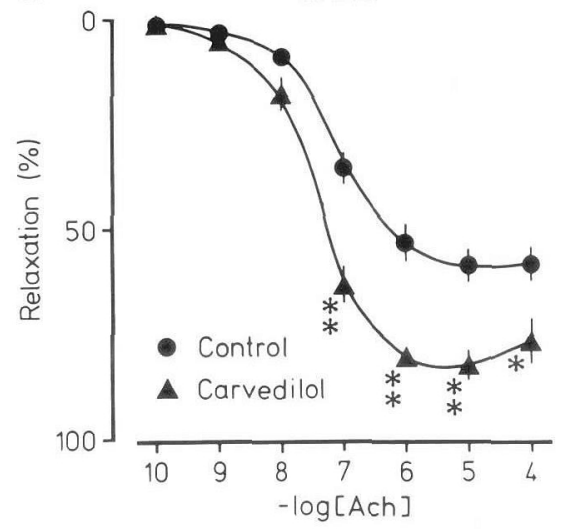

b

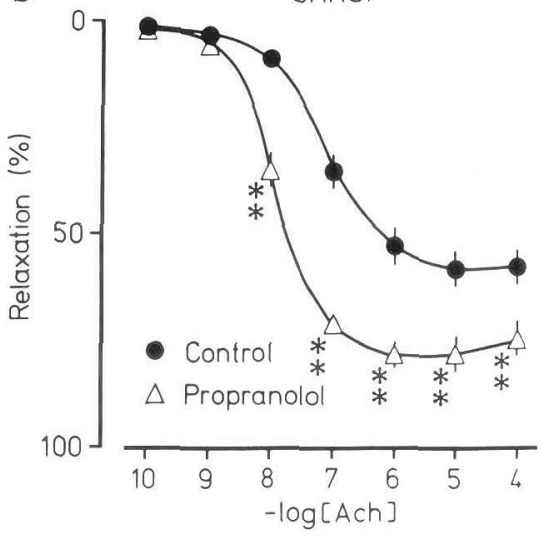

Fig. 8. Effects of treatment of SHRSP with carvedilol (a) and propranolol (b) on acetylcholineinduced relaxation in endothelium-intact preparation. The experiment was performed and expressed same as described in Fig. 7. $\mathrm{n}=18,12$ and 8 in control, carvedilol treated and propranolol treated SHRSP respectively. Asterisks indicate significant differences from control $\left(^{*}, \mathrm{P}<0.05^{* *}, \mathrm{P}<0.01\right)$.
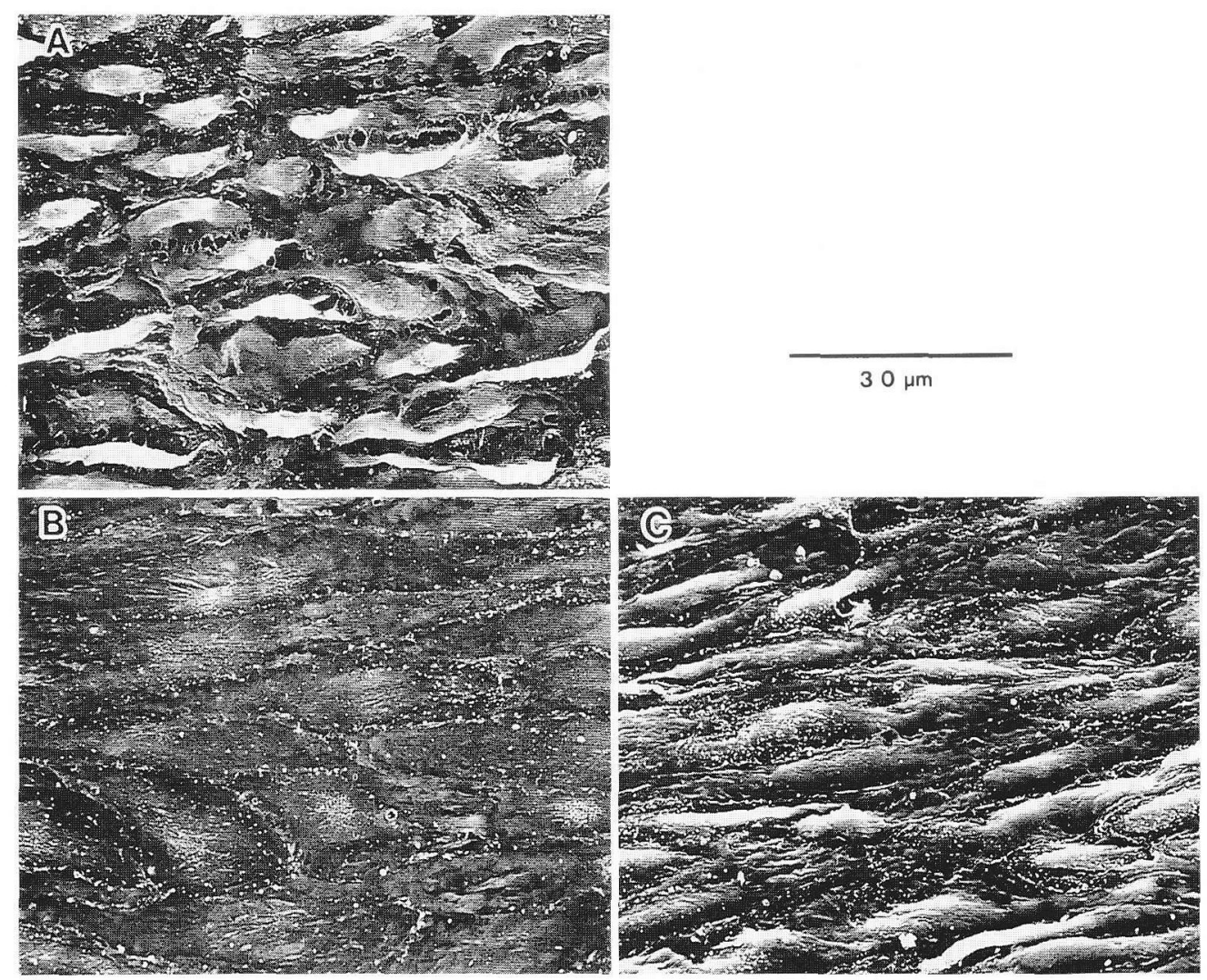

Fig. 9. Scanning electron-micrographs of endothelial surface of aorta of untreated SHRSP (A), carvedilol-treated SHRSP (B) and propranolol-treated SHRSP (C). The treatments of the rats were same as described in the methods. Note the rough surface with crater formation in the endothelium of SHRSP and their protection by the treatments. 
induced contraction of SHRSP aorta did not differ markedly from that of WKY aorta, although the sensitivity to lower concentration of $\mathrm{K}^{+}$was increased slightly in SHRSP (data not shown). Treatment of SHRSP with carvedilol decreased the high- $\mathrm{K}^{+}$-induced contraction slightly (Fig. 6a). The removal of endothelium increased the amplitude of the contraction. Carvedilol treatment did not change the contraction significantly in the endothelium-removed preparation (Fig. 6b). Similar effects were observed by the treatment with propranolol.

\section{Endothelium-dependent relaxation}

Endothelium-dependent relaxation was observed by applying acetylcholine in the preparation precontracted with noradrenaline of submaximal concentration $\left(5 \times 10^{-7} \mathrm{M}\right)$. Acetylcholine relaxed the contraction dose-dependently. The relaxations were abolished by the removal of endothelium or in the presence of $\mathrm{N}^{\mathrm{G}}$-nitro-L-arginine in the preparations both from SHRSP and WKY. The acetylcholine-induced relaxation was significantly smaller in the aorta from SHRSP when compared with that in the aorta from WKY. The threshold concentrations of acetylcholine for the relaxation were not different (Fig. 7).

Treatment with carvedilol caused an enhancement of the acetylcholine-induced relaxation in SHRSP. The threshold concentration for the relaxation was not altered by the treatment. Similar effects were observed by the treatment with propranolol (Fig. 8).

\section{Structural changes of endothelium}

Endothelial surface of the preparation from 16 weeks old SHRSP was rough, and protrusion toward the lumen and crater formation were often observed. Treatment with carvedilol or propranolol prevented these abnormal changes and kept the surface flat and smooth (Fig. 9).

\section{Discussion}

The smooth muscle of aorta from SHRSP exhibited spontaneous tension development (tone) in the absence of stimulant as has been reported in SHR arterial smooth muscle (Noon et al., 1978; Lindner and Heinle, 1987; Fitzpatrick and Szentivanyi, 1980). The tone is thought to be brought about by $\mathrm{Ca}^{++}$influx through voltage-dependent $\mathrm{Ca}^{++}$channel of the smooth muscle membrane, since it was abolished by the removal of extracellular $\mathrm{Ca}^{++}$or by the application of a Ca-antagonist, verapamil. We have observed that intracellular $\mathrm{Ca}^{++}$level is elevated in this preparation (Sasaki et al., 1993) as has also been reported in cultured smooth muscle cells of SHR aorta (Sugiyama et al., 1986, 1990). Above hypothesis is supported also by the evidence that $\mathrm{Ca}^{++}$dependency of skinned smooth muscle is not altered in arteries of spontaneously hypertensive rats including SHRSP (Nghiem and Rapp, 1983 ; Mrwa et al., 1986 ; Dohi et al., 1990). The tone is easily observed in endothelium-removed preparation. Endothelium-derived relaxing factor (EDRF) or endothelium-derived hyperpolarizing factor (EDHF) would depress the tone (Martin, 1988 ; Suzuki and Chen, 1990 ; Lüscher and Vanhoutte, 1990).

It has been reported that the development of the tone in vascular smooth muscle of hypertensive rats can be prevented by treating the rat with angiotensin converting enzyme (ACE) inhibitor or by hydralazine (Sada et al., 1989a, 1989b ; Sunano and Shimamura, 1991; 
Sunano et al., 1992). In the present study, we observed that the development of the tone could also be prevented by the treatment with carvedilol or propranolol.

Noradrenaline-induced contractions of the endothelium-removed preparation indicated that the adrenoceptor of the smooth muscle were not greatly different between the preparation from SHRSP and WKY. In endothelium-intact preparation, on the other hand, marked difference was observed in the contraction. Endothelium depressed the noradrenaline-induced contraction in preparations both from SHRSP and WKY, however, the depression was more remarkable in the preparation from WKY (Osugi et al., 1990; Sunano et al., 1992). This result may indicate that release of EDRF by adrenergic agonists (Martin, 1986, 1988 ; Miller et al., 1988 ; Kaneko and Sunano, 1993) was decreased in the aorta of SHRSP.

The EDRF released spontaneously or released by noradrenaline is thought to be nitric oxide (NO) (Kaneko and Sunano, 1993). Chronic treatment with carvedilol depressed noradrenaline-induced contraction and shifted the dose-response curve to the right in endotheliumintact preparation but not in endothelium-removed preparation. It can therefore be assumed that the depression of noradrenaline-induced contraction by the treatment is due to the change in the function of endothelium and not in that of smooth muscle. Thus, carvedilol-treatment could depress the noradrenaline-induced contraction in endothelium-dependent manner.

Treatment of SHRSP with carvedilol or propranolol depressed the dose-response curve for high- $\mathrm{K}^{+}$-induced contraction of endothelium-intact preparation slightly. No influence of the treatment was observed in endothelium-removed preparation. These may indicate that the treatments did not affect activation of voltage-dependent $\mathrm{Ca}$ channels.

The endothelium-dependent relaxation has been shown to be impaired in aorta of spontaneously hypertensive rats (Konishi and Su, 1983; Winquist, 1988; Sunano et al., 1989 ; Lüscher et al., 1991). We also ascertained that the acetylcholine-induced endothelium-dependent relaxation was impaired in SHRSP aorta. The prevention of the impairment of endotheliumdependent relaxation by antihypertensive treatment has been reported with hydralazine (Sunano et al., 1989; Shimamura et al., 1991; Sunano et al., 1993) and ACE inhibitors (Clozel et al., 1990 ; Clozel, 1991 ; Shimamura et al., 1991 ; Sunano et al., 1992), where blood pressure of hypertensive rats was kept as low as that of normotensive rats. Present results that carvedilol-treatment prevented the impairment of the relaxation indicate that the impairment could also be prevented by the antihypertensive treatment with adrenergic blocking agents. It is noteworthy that the similar degree of endothelium-dependent relaxation to that of WKY aorta could be obtained in the aorta from carvedilol- or propranolol-treated SHRSP, although blood pressure was kept similar to that of SHR in which endothelium-dependent relaxation has been reported to be impaired (Konishi and Su, 1983 ; Sugimoto et al., 1988 ; Sunano et al., 1989 ; Lüscher et al., 1991). Thus, the possibility of the involvement of other factors such as adrenergic receptors in the improvement of the endothelium-dependent relaxation was suggested, although further studies were required to reach a conclusion.

Endothelium of the arteries of spontaneously hypertensive rats has been known to be structurally altered (Hazama et al., 1979; Limas et al., 1980 ; Lüscher and Vanhoutte, 1990 ; Sunano et al., 1993). The impairment of endothelium-dependent relaxation in SHR and SHRSP aorta was accompanied with the structural abnormalities of the endothelium as has 
been reported in arteries of SHR (Lüscher, 1988; Sunano et al., 1993). The structural abnormalities of the endothelium can be prevented by antihypertensive treatment (Limas et al., 1983 ; Sunano et al., 1993). In the present experiment, it was demonstrated that the abnormalities could be prevented by the treatment with carvedilol or propranolol. As the blood pressure of SHRSP treated by carvedilol or propranolol was kept much higher than that of WKY, this also suggests the possibilities of involvement of adrenergic receptor in preventing structural abnormalities of endothelium.

In conclusion, chronic antihypertensive treatment with carvedilol decreased spontaneous muscle tone and noradrenaline-induced contraction while increased acetylcholine-induced relaxation in SHRSP aorta. As endothelium as well as smooth muscle was responsible for these changes, these results indicated carvedilol-treatment affected both endothelium and smooth muscle. All of these changes may play roles in lowering blood pressure of SHRSP under carvedilol-treatment.

\section{References}

Clozel, M. (1991). Mechanism of action of angiotensin converting enzyme inhibitors on endothelial function in hypertension. Hypertension 18(suppl. II) : II-37-II-42.

Clozel, M., Kuhn, H. and Hefti, F. (1990). Effects of angiotensin converting enzyme inhibitors and hydralazine on endothelial function in hypertensive rats. Hypertension 16: 532-540.

Dohi, Y., Aoki, K., Fujimoto, S., Kojima, M. and Matsuda, T. (1990). Alteration in sarcoplasmic reticulum-dependent contraction of tail arteries in response to caffeine and noradrenaline in spontaneously hypertensive rats. J. Hypertension 8: 261-267.

Fitzpatrick, D.F. and Szentivanyi, A. (1980). The relationship between increased myogenic tone and hyporesponsiveness in vascular smooth muscle of spontaneously hypertensive rats. Clin. Exp. Hypertension 2: 1023-1037.

Fujii, K., Tominaga, M., Ohmori, S., Kobayashi, K., Koga, T., Takata, Y. and Fujishima, F. (1992). Decreased endothelium-dependent hyperpolarization to acetylcholine in smooth muscle of the mesenteric artery of spontaneously hypertensive rats. Circ. Res. 70:660-669.

Hashimoto, H., Tanaka, M., Kanda, A. and Akashi, A. (1988). Analysis of the mechanism underlying the vasodilator action of carvedilol in pithed spontaneously hypertensive rats. Drugs 36 (Suppl. 6) : 31-36.

Hashimoto, H., Kanda, A. and Kubo, H. and Tanaka, M. (1991). Features of the acute hypotensive action of carvedilol and its ameliorating effect on myocardial ischemia. J. Cardiovasc. Pharmacol. 18 (Suppl. 4) : S22-S28.

Hazama, F., Ozaki, T. and Amano, S. (1979). Scanning electron microscopic study of endothelial cells of cerebral arteries from spontaneously hypertensive rats. Stroke $10: 245-252$.

Kaneko, K. and Sunano, S. (1993). Involvement of $\alpha$-adrenoceptors in the endothelium-dependent depression of noradrenaline-induced contraction in rat aorta. Eur. J. Pharmacol. 240 : 195200 .

Konishi, M. and Su, C. (1983). Role of endothelium in dilator responses of spontaneously hypertensive rat arteries. Hypertension 5 : 881-886.

Limas, C., Westrum, B. and Limas, C.J. (1980). The evolution of vascular changes in the spontaneously hypertensive rat. Am. J. Pathol. 98 : 357-384.

Limas, C., Westrum, B. and Limas, C.J. (1983). Effect of antihypertensive therapy on the vascular changes of spontaneously hypertensive rats. Am. J. Pathol. 111 : 380-393. 
Lindner, V. and Heinle, H. (1987). $\mathrm{Ca}^{2+}$ influx in spontaneously hypertensive rats is sensitive to calcium antagonists. Eur. J. Pharmacol. 138: 147-149.

Lüscher, T.F. (1988). Endothelial vasoactive substances and cardiovascular disease. Karger, Basel.

Lüscher, T.F. and Vanhoutte, P.M. (1990). The endothelium: Modulator of Cardiovascular function. CRC Press, Boca Raton.

Lüscher, T.F., Dohi, Y., Tanner, F.C. and Boulanger, C. (1991). Endothelium-dependent control of vascular tone: effects of age, hypertension and lipids. Basic Res. Cardiol. 86(Suppl. 2) : 143158.

Martin, W., Furchgott, R.F., Villani, G.M. and Jothanandan, D. (1986). Depression of contractile responses in rat aorta by spontaneously released endothelium-derived relaxing factor. $J$. Pharmacol. Exp. Ther. 237 : 529-538.

Martin, W. (1988). Basal release of endothelium-derived relaxing factor. In: Relaxing and contracting factors. : ed. by P.M. Vanhoutte, Humana Press, Clifton, NJ, pp. 159-178.

McTavish, D., Campoli-Richards, D. and Sorkin, E.M. (1993). Carvedilol: A review of its pharmacodynamic and pharmacokinetic properties and therapeutic efficacy. Drugs 45:232-258.

Miller, R.C., Schini, V. and Schoeffter, P. (1988). Modulation by the endothelium of agonist-induced contractions of vascular smooth muscle. In : Relaxing and contracting factors, ed. by P.M. Vanhoutte, Humana Press, Clifton, NJ, pp. 241-265.

Mrwa, U., Guth, K., Haist, C., Troschka, M., Herrmann, R., Wojciechowski, R. and Gagelmann, M. (1986). Calcium-requirement for activation of skinned vascular smooth muscle from spontaneously hypertensive (SHRSP) and normotensive control rats. Life Sci. 38: 191-196.

Nghiem, C.X. and Rapp, J.P. (1983). Responses to calcium of chemically skinned vascular smooth muscle from spontaneously hypertensive rats. Clin. Exp. Hypertension A5 : 849-856.

Nigro, D., Bruno, Z., Carvalho, M.H.C. and Scivoletto, R. (1989). Chronic but not acute treatment with hydralazine reserves the decreased endothelium-dependent response in spontaneously hypertensive rats. Clin. Exp. Hypertension A11: 573-586.

Noon, J.P., Rice, P.J. and Baldessarini, R. (1978). Calcium leakage as a cause of the high resting tension in vascular smooth muscle from the spontaneously hypertensive rat. Pro. Nat. Acad. Sci. (USA) $75:$ 1605-1607.

Okamoto, K., Yamori, Y. and Nagaoka, A. (1974). Establishment of the stroke-prone spontaneously hypertensive rat (SHR). Circ. Res. 34/35 suppl. I : I-143-153.

Osugi, S., Shimamura, K. and Sunano, S. (1990). Decreased modulation by endothelium of noradrenaline-induced contractions in aorta from stroke-prone spontaneously hypertensive rats. Arch. Int. Pharmacody. 305: 86-99.

Prichar, B.N.C. (1992). $\beta$-blocking agents with vasodilating action. J. Cardiovasc. Pharmacol. 19(Suppl. 1) : S1-S4.

Rahn, K.H. (1987). Antihypertensive compounds with combined actions. J. Cardiovasc. Pharmacol. 10 (Suppl. 11) : S18-S22.

Sada, T., Koike, H., Nishio, H. and Oizumi, K. (1989a). Chronic inhibition of angiotensin converting enzyme decreases $\mathrm{Ca}^{2+}$-dependent tone of aorta in hypertensive rats. Hypertension 13: 582588.

Sada, T., Koike, H. and Miyamoto, M. (1989b). Long-term inhibition of angiotensin converting enzyme suppresses calcium channel agonist-induced contraction of aorta in hypertensive rats. Hypertension 14: 652-659.

Sasaki, F., Osugi, S., Shimamura, K. and Sunano, S. (1993). Relationship between blood pressure and smooth muscle tone in aortae of hypertensive rats: roles of $\left[\mathrm{Ca}^{2+}\right]$. J. Smooth Muscle Res. 29 : $69-79$.

Seki, N., Nagao, T., Komori, K. and Suzuki, H. (1988). Alpha and beta-adrenoceptor blocking action of carvedilol in the canine mesenteric artery and vein. J. Pharmacol. Exp. Ther. 246 : 
$1116-1122$

Shimamura, K., Osugi, S., Moriyama, K. and Sunano, S. (1991). Impairment and protection of endothelium-dependent relaxation in aortae of various strains of spontaneously hypertensive rats. J. Cardiovasc. Pharmacol. 17 (Suppl. 3) : S133-S136.

Sponer, G., Bartsch, W., Strein, K., Müller-Beckmann, B. and Bohm, E. (1987). Pharmacological profile of carvedilol as a $\beta$-blocking agent with vasodilating and hypotensive properties. $J$. Cardiovasc. Pharmacol. 9: 317-327.

Sugimoto, T., Tobian, T. and Ganguli, M.C. (1988). High potassium diets protect against dysfunction of endothelial cells in stroke-prone spontaneously hypertensive rats. Hypertension 11 : 579 585.

Sugiyama, T., Yoshizumi, M., Takaku, F., Urabe, H., Tsukakoshi, M., Kasuya, T. and Yazaki, Y. (1986). The elevation of the cytoplasmic calcium ions in vascular smooth muscle cells in SHR-measurement of the free calcium ions in single living cells by lasermicro-fluorospectrometry. Biochem. Biophysic. Res. Comm. 141: 340-345.

Sugiyama, T., Yoshizumi, M., Takaku, F. and Yazaki, Y. (1990). Abnormal calcium handling in vascular smooth muscle cells of spontaneously hypertensive rats. J. Hypertension 8 : 369375.

Sunano, S. and Shimamura, K. (1991). Smooth muscle tone of aorta of spontaneously hypertensive rats due to an increase in voltage dependent $\mathrm{Ca}$ channel and its modification by endothelium. In: Ion channels of vascular smooth muscle cells and endothelial cells, ed. by N. Sperelakis and H. Kuriyama, Elsevier, New York, pp. 225-233.

Sunano, S., Osugi, S. and Shimamura, K. (1989). Blood pressure and impairment of endotheliumdependent relaxation in spontaneously hypertensive rats. Experientia 45 : 706-708.

Sunano, S., Osugi, S., Kaneko, K., Yamamoto, K. and Shimamura, K. (1992). Effects of chronic treatment with SQ29852 on spontaneous smooth muscle tone and endothelium-dependent relaxation in aorta of stroke-prone spontaneously hypertensive rats. J. Cardiovasc. Pharmacol. 19 : 602-609.

Sunano, S., Itoh, H., Isogai, N., Osugi, S., Yamamoto, K. and Shimamura, K. (1993). Effects of antihypertensive treatment on endothelial structure and endothelium-dependent relaxation in aorta of stroke-prone spontaneously hypertensive rats. J. Vasc. Med. Biol. 4: 235-242.

Suzuki, H. and Chen, G. (1990). Endothelium-derived hyperpolarizing factor (EDHF) : an endogeneous potassium-channel activator. News Physiol. Sci. 5 : 212-215.

Triggle, C.R. and Laher, I. (1985). A review of changes in vascular smooth muscle functions in hypertension: isolated tissue versus in vivo studies. Can. J. Physiol. Pharmacol. 63 : 355-365.

Winquist, R.J. (1988). Endothelium-dependent relaxations in hypertensive blood vessels. In: Relaxing and contracting factors, ed. by P.M. Vanhoutte, Humana Press, Clifton, NJ, pp. 473509. 